\title{
LncRNA EGOT/miR-2I I-5p Affected Radiosensitivity of Rectal Cancer by Competitively Regulating ErbB4
}

\author{
Chunxiang $\mathrm{Li}^{1}$ \\ Hengchang Liu ${ }^{2}$ \\ Ran $\mathrm{Wei}^{2}$ \\ Zheng Liu ${ }^{2}$ \\ Haipeng Chen ${ }^{2}$ \\ Xu Guan ${ }^{2}$ \\ Zhixun Zhao ${ }^{2}$ \\ Xishan Wang ${ }^{2}$ \\ Zheng Jiang ${ }^{2}$
}

'Department of Thoracic Surgery, National Cancer Center/National Clinical Research Center for Cancer/Cancer Hospital, Chinese Academy of Medical Sciences and Peking Union Medical College, Beijing, People's Republic of China; ${ }^{2}$ Department of Colorectal Surgery, National Cancer Center/ National Clinical Research Center for Cancer/Cancer Hospital, Chinese Academy of Medical Sciences and Peking Union Medical College, Beijing, People's Republic of China
Background/Aims: Long non-coding ribonucleic acids (lncRNAs) are involved in the progression of cancers and affect the response to radiation therapy. This study was to investigate the mechanism of lncRNA EGOT in the radiosensitivity of rectal cancer.

Methods: The mRNA expression of EGOT, miR-211-5p and ErbB4 in rectal cancer tissues and cells was detected by qRT-PCR. The protein expression of ErbB4 was detected by Western blot. Dual-luciferase reporter assay and ribonucleic acid immunoprecipitation (RIP) were used to confirm the interaction between EGOT and miR-211-5p or miR-211-5p and ErbB4. Transfection technology was used to down-regulate and up-regulate the expression of EGOT and miR-211-5p in rectal cancer cells, respectively. MTT, colony formation and flow cytometry were used to detect the effect of EGOT and miR-211-5p on proliferation, invasion, migration and apoptosis of rectal cancer cells.

Results: The expression of EGOT was up-regulated in rectal cancer tissues and cells, and the expression of EGOT was related to the late stage of pathology. EGOT knockdown inhibited the proliferation and colony formation of rectal cancer cells and induced the apoptosis of rectal cancer cells. Moreover, EGOT knockdown was significantly enhanced the effects of radiotherapy on rectal cancer in vivo and in vitro. Furthermore, EGOT was found to serve as a sponge of miR-211-5p, and ErbB4 was a downstream target of miR-211-5p. EGOT enhanced the expression of ErbB4 by regulating miR-211-5p. MiR-211-5p inhibitor restored the effect of EGOT knockdown on the radiosensitivity of rectal cancer.

Conclusion: Down-regulation of EGOT could inhibit the growth of rectal cancer cells by regulating the miR-211-5p/ErbB4 axis and improve the radiosensitivity of rectal cancer cells. EGOT may be a new therapeutic target for rectal cancer.

Keywords: EGOT, miR-211-5p, ErbB4, rectal cancer, radiosensitivity

\section{Introduction}

Rectal cancer is one of the most common malignant tumors. It currently ranks third in the world's most common cancer and the fourth most common cause of cancer-related death in the world. ${ }^{1}$ Due to the improvement of living standards and changes in environment and lifestyle, the incidence rate and risk factors also increased, and the age of onset is becoming younger. ${ }^{2}$ Because the early symptoms of rectal cancer in the population are not obvious, many patients were found in the physical examination, but are already in the late stage. ${ }^{3,4}$ The current clinical treatments mainly include surgical resection, radiotherapy, chemotherapy and immunotherapy. ${ }^{5}$ Although the diagnosis and treatment are satisfactory, the prognosis is still not optimistic, and about two-thirds
Correspondence: Zheng Jiang

Department of Colorectal Surgery, National Cancer Center/National Clinical Research Center for Cancer/Cancer Hospital, Chinese Academy of Medical Sciences and Peking Union Medical College, Chaoyang District, Beijing, People's Republic of China

Tel +86 10-87787। I0

Email zr7780@।63.com 
of rectal cancer patients have recurrence and distant metastasis. ${ }^{6}$ At present, radiation therapy is considered to be the important treatments for rectal cancer, especially for locally advanced patients. ${ }^{7}$ Unfortunately, radiotherapy resistance is one of the main reasons for the failure of rectal cancer treatment, and its molecular mechanism has not been fully understood. ${ }^{8}$ Therefore, in order to further improve the effect of radiotherapy, it is necessary to investigate the molecular mechanism of radiotherapy resistance of rectal cancer.

In the human genome sequence, most of the products are non-coding transcripts during the transcription process. Non-coding RNA (ncRNA) refers to RNA sequences that are transcribed by genes but are not translated into proteins in the end. ${ }^{9}$ In recent years, the regulation of IncRNA has been sought after by many tumor researchers. ${ }^{10}$ Studies have shown that IncRNA is closely related to various tumor behaviors of malignant tumors, such as tumor autophagy, tumor resistance and tumor immunity. ${ }^{11}$ In the field of malignant tumors, lncRNA expression disorders can also cause changes in their biological behavior and even tumor radiosensitivity. ${ }^{12}$ The IncRNA eosinophil granule ontogeny transcript (EGOT) is located at 3p26.1. The previous study revealed that EGOT was downregulated in breast cancer, and its expression was associated with malignant status and poor prognosis. ${ }^{13}$ In addition, next-generation deep sequencing revealed that EGOT might be functional in clear cell carcinoma. ${ }^{14}$ For example, EGOT promotes the occurrence of gastric cancer through the Hedgehog pathway. ${ }^{15}$ However, the effect of EGOT on rectal cancer and radiosensitivity has not been reported.

LncRNAs regulate tumor resistance by many mechanisms, which can affect mRNA expression or competitively bind miRNA to affect the downstream target genes. ${ }^{16}$ miRNA is a kind of non-coding RNA but can play a similar coding role by regulating coding genes. miRNA plays a key role in various areas of cells, including cell proliferation, apoptosis, migration, invasion and differentiation. ${ }^{17}$ miRNA can activate or silence some oncogenes or cancer suppressors. ${ }^{18}$ Studies have shown that the abnormal expression of miRNA is closely related to tumor development and radiosensitivity. ${ }^{19}$ MiR-211-5p is involved in proliferation, migration and invasion of certain tumors. ${ }^{20}$ For example, miR-211-5p is involved in the resistance of BRAF in melanoma. ${ }^{21}$ It has been found that the up-regulation or down-regulation of miRNAs can directly cause the abnormal expression level of target gene, and ultimately changes the drug sensitivity of tumor cells through cell signaling pathway. ${ }^{22}$ The ErbB4 receptor is the fourth epidermal growth factor receptor in humans, which belongs to a member of the epidermal growth factor receptor family and has different expressions in normal human tissues. ${ }^{23}$ ErbB4 is overexpressed in a variety of tumor tissues, suggesting that it plays an important role in the development of tumors. ${ }^{24}$ This study was to investigate the mechanism of EGOT in the radiosensitivity of rectal cancer and try to provide a new therapeutic target for rectal cancer.

\section{Materials and Methods}

\section{Tissue Sample}

The pairs of tumors and adjacent normal tissues of patients with rectal cancer were collected from Chinese Academy of Medical Sciences and Peking Union Medical College $(n=40)$. All patients suffered from primary nonmetastatic rectal cancer and received complete surgical resection in Chinese Academy of Medical Sciences and Peking Union Medical College after providing informed written consent and agreement. According to the National Comprehensive Cancer Network Guidelines for Esophageal Cancer, normal tissue was at least $5 \mathrm{~cm}$ from the primary lesion. This study was approved by the Chinese Academy of Medical Sciences and Peking Union Medical College.

\section{Cell Culture and Transfection}

Human rectal cancer cell lines (HR8348 and Colo320) and human normal colon cell line NCM460 were purchased from Cell Center of Shanghai Institute of Biological Sciences. Cells were cultured in DMEM medium containing $10 \%$ fetal bovine serum (FBS). Cells were incubated in a $5 \% \mathrm{CO}_{2}$ cell incubator at $37^{\circ} \mathrm{C}$. The radioresistant human rectal cancer cell line Colo320 (radioresistant Colo320) was obtained, and the Colo320 cells were screened by dose gradients. The radiosensitivity of Colo320 was verified by clonogenesis experiment.

The cells at logarithmic growth stage were transfected, and the fusion rate was $60 \%$. Si-EGOT, miR-211-5p mimics, miR-211-5p agomir, and their respective controls (Gene Pharma, Shanghai, China) were transfected into the cells with Lipofectamine 2000 (Invitrogen, Waltham, Ma, USA). To construct EGOT, ErbB4 overexpression plasmids, their sequences were cloned into pCDS-At cloning vectors (BioVector, Beijing, China). Oligonucleotides 
were transfected into cells by Lipofectamine 2000 (Invitrogen, Carlsbad, CA).

\section{Cell Proliferation Detection}

Cells were seeded in a 96-well plate at a density of 50,000 cells per well. $100 \mu \mathrm{L}$ of CCK8 solution (Liji, Shanghai, China) was added to each well. After $4 \mathrm{~h}$ of incubation, absorbance value was measured by a microplate reader (Bio Tek Instruments, USA) at $450 \mathrm{~nm}$.

\section{Colony Formation Assays}

Cells were seeded into 6-well plates with or without 2 Gy radiation. After two weeks, cells were fixed and stained with $0.1 \%$ crystal violet solution. The colonies were counted under an inverted microscope.

\section{Transwell Analysis}

The cells were seeded overnight and treated for $48 \mathrm{~h}$. The migration experiments were performed using a 24-well Transwell system (Costar, Badhoevedorp, Netherlands). The cell suspension was maintained in the upper cavity of the Transwells and then subjected to matrigel invasion test.

\section{Flow Cytometry}

Cells were plated in 6-well plates at a density of $5 \times 10^{5}$ cells/well, and the cells were collected and counted when the cells grew to the logarithmic growth phase. The cells were centrifuged and $195 \mu \mathrm{L}$ of Annexin V-FITC binding solution was added to resuspend the cells. $5 \mu \mathrm{L}$ Annexin V-FITC and $10 \mu \mathrm{L}$ propidium iodide staining solution were added. Then, the cells were incubated in the dark for 10-20 min and then placed in an ice bath.

\section{Quantitative Reverse Transcription PCR (qRT-PCR)}

Total RNA in tissues and cells was extracted using TRIzol reagent (Invitrogen, Carlsbad, CA). The quality of RNA was analyzed using NanoDrop 1000 (NanoDrop Technologies; Thermo Fisher Scientific, Inc.). SYBRGreen (Takara Biotechnology, Co., Lt., Dalian, China) was used in the RT-PCR reaction. Amplification was performed on an ABI 7500 real-time PCR system. A qScript microRNA cDNA synthesis kit (Quantabio, Beverly, California, USA) was used for cDNA synthesis. The $2^{-\triangle \Delta C T}$ method was used to calculate the expression levels of EGOT, miR-211-5p and ErbB4. The expression levels of miRNA were normalized by U6, respectively. The primer sequences were as follows:

LncRNA EGOT forward 5'- CTAATCCACTTGGCC GGAG-3',

LncRNA EGOT reverse 5'- CGCCTAGATTCCAA AGG-3'.

miR-211-5p forward: 5'- CTGAATGTGAGGAGG ATGT-3',

miR-211-5p reverse: 5'-GTCCTTCGGCATCCCGG CCG-3'.

ErbB4: forward 5'-CAACGGATTCCGCGGCCCT-3', ErbB4 reverse: 5'-GAATTCTTACCAGCCTTGGC-3'. GAPDH forward: 5'-CGAGAGAATCCGCGGA CAT-3',

GAPDH reverse: 5'-TTGTGCAATACAGCGTG GAC-3'.

U6 forward: 5'-GACAGATTCGGTCTGTGGCAC-3', U6 reverse: 5'-GATTACCCGTCGGCCATCGATC-3' .

\section{Western Blot}

The transfected cells were collected, and the total protein was extracted. The protein concentration was quantified by the BCA Protein As-say Kit and transferred to the PVDF membrane by electrophoresis. The membrane was incubated with anti-ErB4 (abcam, 1: 1000, UK) and antiGAPDH antibodies (1: 1000, Abcam, Cambridge, UK) for overnight at $4{ }^{\circ} \mathrm{C}$, followed by incubation with antirabbit secondary antibody (1: 1000, Cell Signaling Technology, Boston, MA, USA) for $1 \mathrm{~h}$. Then, the protein was visualized in an enhanced chemiluminescence reaction solution (Pierce, Rockford, IL). The experimental methods of Western blot were performed with reference. ${ }^{25}$

\section{Luciferase Reporter Assay}

The transcriptome expression data in read were downloaded from the TCGA database, and the expression level of lncRNA was extracted. According to the clinical information of the samples, 22 samples received radiotherapy and 112 samples did not receive radiotherapy. The differences between the two groups were analyzed, and $155 \operatorname{lncRNAs}$ with significant difference were selected according to " $p$ value $<0.05 \&|\operatorname{logFC}|>1$ ". The top 50 of differential lncRNA expression was extracted to draw the cluster heat map of differential lncRNA expression. The results were shown in Supplementary Figure 1. Wild-type or mutant of circBIRC6 was subcloned into the pmirGLO vector. PmirGLO, pmirGLO-EGOT or pmirGLO-EGOTmut with miR-211-5p mimic were co-transfected into cells 
by Lipofectamine 2000 (Invitrogen). After 48 h, luciferase activity was measured using a dual luciferase assay kit (Promega).

\section{Nude Mouse Tumor Xenograft Model}

Male athymic BALB/c nude mice were purchased from the National Laboratory Animal Center (Beijing, China). First, a rectal cancer xenograft model was established by subcutaneous injection of Colo320 cells. A single X-ray dose of 2Gy was used to irradiate Colo320 mice subcutaneously Xenograft model. In addition, a total of $5 \times 10^{6}$ shEGOT-Colo320 cells and NC-Colo320 cells were suspended in $100 \mu \mathrm{L}$ phosphate buffer solution and then injected subcutaneously into nude mice. Five weeks later, mice were euthanized. The study has been approved by the Ethics Committee of Chinese Academy of Medical Sciences and Peking Union Medical College and experiments had been performed in accordance with the National Institute of Health Laboratory Animal Care and Use Guidelines.

\section{RNA Pull-Down Assay}

RNA immunoprecipitation assay was performed using EZMagna RIP kit (Millipore). Cells were lysed using complete RNA immunoprecipitation (RIP) lysis buffer. Cell extracts were incubated with magnetic beads conjugated with anti-Argonaute 2 (ago2) or control antiimmunoglobulin $\mathrm{G}$ (IgG) antibodies for $6 \mathrm{~h}$. Then, as protein beads were removed, the RNA was purified by RTqPCR analysis.

\section{Immunofluorescence Analysis of $\gamma-\mathrm{H} 2 \mathrm{AX}$}

The specific methods and steps are as follows: (1) slowly add $7 \mathrm{~mL}$ diluted blood sample to $7 \mathrm{~mL}$ Ficoll separation solution, pay attention to keep the stratification interface clear, centrifuge treatment; (2) The PBMCs were prepared into cell suspensions with 1640 medium for $7 \mathrm{~min} \times 2$ times, and the samples were prepared on the slide; (3) the specimens were fixed with $4 \%$ paraformaldehyde for 15 min; (4) PBS solution was used for $5 \mathrm{~min} \times 3$ times; $0.2 \%$ triton-x 100 was used to break the membrane for $15 \mathrm{~min}$, and then eluted with PBS solution for $5 \mathrm{~min} \times 3$ times; (5) Goat serum working solution was used to seal for $1 \mathrm{~h}$; (6) Mouse anti gamma $\mathrm{H} 2 \mathrm{AX}$ antibody diluted at $1: 150$ (upstate company, USA) was added and overnight at $4^{\circ} \mathrm{C}$ in a wet box; and the Goat anti mouse IgG secondary antibody diluted at 1:350 (Molecular Probes company, USA) was added for $1 \mathrm{~h}$ at room temperature, and then eluted with $1 \%$ PBST for $10 \mathrm{~min} \times 4$ times; 6 -diaminol2-phenylindole (DAPI) was re stained for $10 \mathrm{~min}$, and then eluted with $1 \%$ PBST for $10 \mathrm{~min} \times 2$ times; and the number of fluorescence spots of $\gamma$-H2AX was observed under fluorescence microscope with $90 \%$ glycerin.

\section{Statistical Methods}

The monitoring data were analyzed by SPSS19.0 statistical software. The results of data analysis were represented as mean \pm standard deviation (mean $\pm \mathrm{SD}$ ). Multigroup data analysis was founded on one-way ANOVA. LSD test was used for subsequent analysis. $P<0.05$ indicated the difference was significant.

\section{Results}

\section{The Expression of EGOT Was}

\section{Up-Regulated in Rectal Cancer Tissues and Cell Lines}

To explore the potential role of EGOT in rectal cancer, the expression of EGOT was analyzed in rectal cancer tissues and cell lines. As shown in Figure 1A, compared with Normal group, the expression of EGOT was significantly increased in rectal cancer tissues $(p<0.05)$. Moreover, we found that the expression levels of EGOT were highest in most rectal cancer tissues compared with Normal group (Figure 1B, $p<0.05$ ). In addition, compared with NCM460 cells, the expression of EGOT in rectal cancer cell lines (Colo320 and HR8348) was significantly increased (Figure 1C, $p<0.05$ ), which was consistent with the results in rectal cancer tissues. Based on the average expression level of EGOT, the rectal cancer tissue was divided into high EGOT and low EGOT groups. As shown in Figure 1D, the overall survival rate of patients in the EGOT high expression group was significantly reduced $(p<0.05)$. These data indicated the potential carcinogenic effect of EGOT in rectal cancer.

\section{Knockdown of EGOT Inhibited Rectal Cancer Growth}

Next, the biological functions of EGOT in the proliferation, invasion and apoptosis of rectal cancer cells were analyzed. As shown in Figure 2A, compared with the si$\mathrm{NC}$ group, the expression of EGOT was significantly reduced in si-EGOT group $(p<0.05)$, indicating that the transfection was successful. Compared with the si-NC group, si-EGOT significantly reduced the viability and the number of colony formation of Colo320 cells 

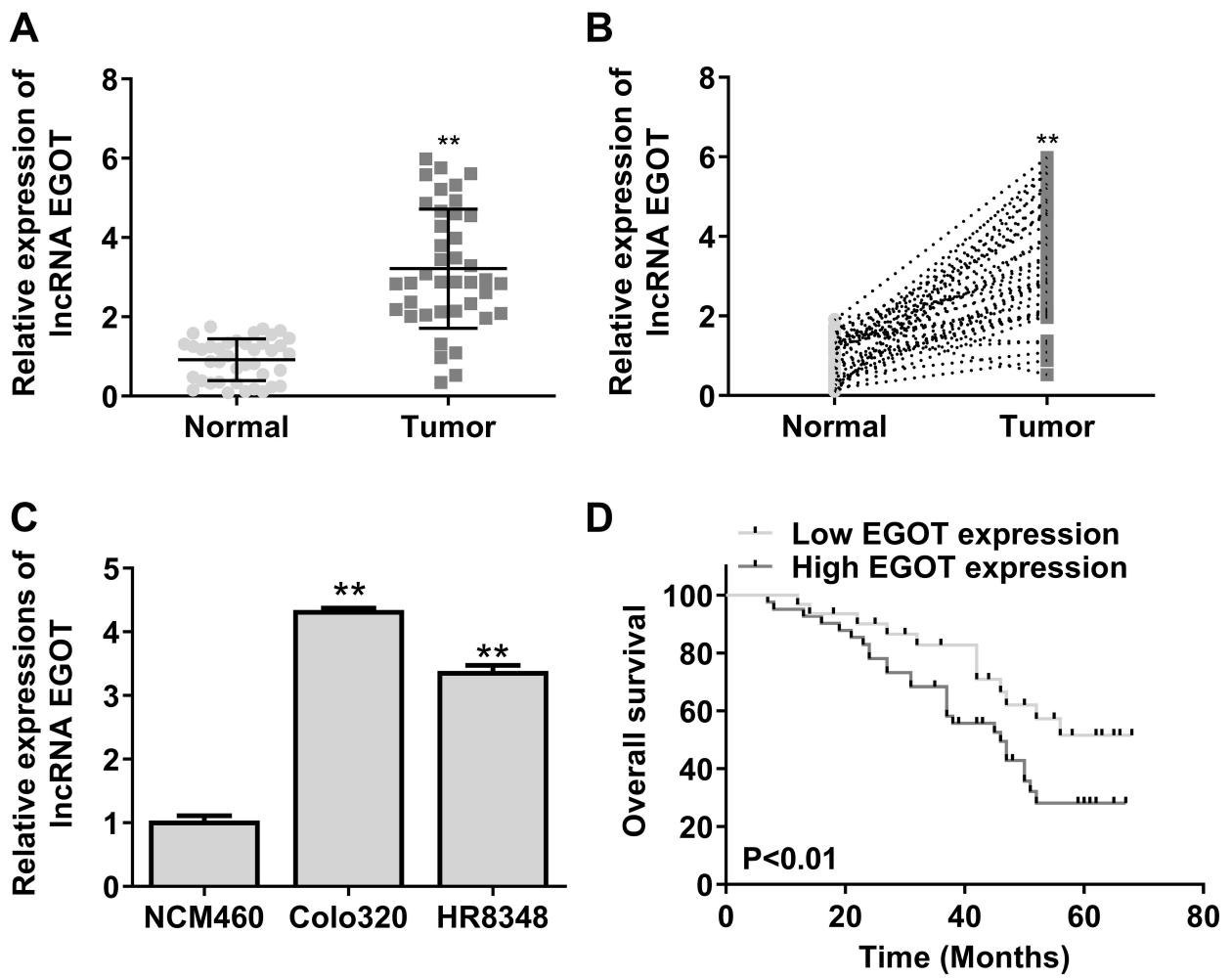

Figure I EGOT expression in rectal cancer tissues and cell lines. (A and B) Detection of EGOT expression in rectal cancer tissues and paired normal tissues by qRT-PCR $(n=50)$. (C) The expression of EGOT in rectal cancer cell lines. (D) Kaplan-Meier survival analysis of EGOT expression. $\mathrm{N}=3$. **p $<0.0 \mathrm{I}$.

( $p<0.05$, Figure 2B and C). In addition, Transwell assay showed that si-EGOT significantly inhibited the invasion and migration of Colo320 cells ( $p<0.05$, Figure 2D) and induced the apoptosis of Colo320 cells $(p<0.05$, Figure 2E). These results showed that EGOT knockdown could inhibit growth of rectal cancer cells.

\section{EGOT Was Involved in Rectal Cancer Radiotherapy and Affected \\ Radiosensitivity}

Next, whether EGOT can affect the radiosensitivity of rectal cancer was analyzed. Colo320 cells were selected and given different irradiation doses (0, 2, 4, 8, 10Gy X-ray). As shown in Figure 3A, the expression of EGOT was significantly increased in a dose-dependent manner after treated with the above irradiation dose $(p<0.05)$. Considering the cell sensitivity, 2Gy was selected for subsequent experiments. As shown in Figure 3B and C, both si-EGOT and 2Gy could significantly inhibit cell proliferation, and the combination treatment of si-EGOT and 2Gy had more significant inhibitory effect on cell proliferation $(p<0.05)$. In addition, both si-EGOT and 2Gy significantly induced apoptosis, and the combined treatment of si-EGOT and 2Gy more significant induced apoptosis (Figure 3D, $p<0.05$ ). These results indicated that EGOT was involved in radiotherapy for rectal cancer and affected radiosensitivity.

\section{EGOT Served as a Sponge of miR-2II-5p}

Next, the potential mechanism of EGOT to regulate the progression of rectal cancer was explored. MiR-211-5p was identified as a potential target of EGOT through online prediction tool Starbase v2.0 (Figure 4A). The luciferase reporter assay showed that luciferase activity was significantly reduced in miR-211-5p mimic and EGOT-WT co-transfected cells $(p<$ 0.05 ), but there was no significant change in luciferase activity of EGOT-MUT (Figure 4B). As shown in Figure 4C, compared with anti-IgG immunoprecipitates, IncRNA EGOT and miR-211-5p were preferably enriched in miRNPs containing Ago2. In addition, compared with si-NC group, the expression of miR-211-5p was significantly reduced in the si-EGOT group ( $p<0.05$, Figure 4D). As shown in Figure 4E, compared with the Normal group, the expression of miR-211-5p was significantly decreased $(p<0.05)(\mathrm{n}=50)$. As shown in Figure $4 \mathrm{~F}$, the expression of EGOT in rectal cancer tissue was negatively correlated with the expression of miR-211-5p $\left(\mathrm{R}^{2}=\right.$ 0.695). These results suggested that EGOT might directly target miR-211-5p in rectal cancer. 


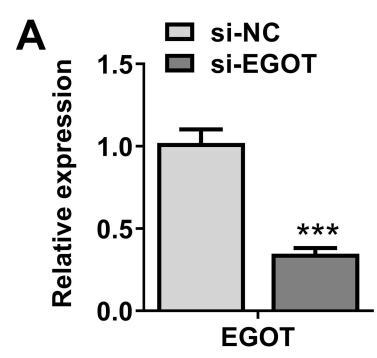

Si-NC

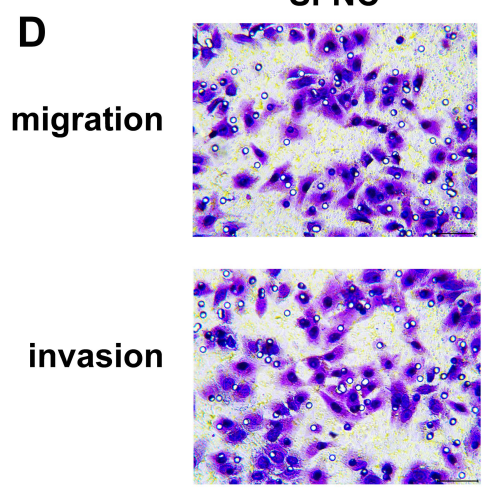

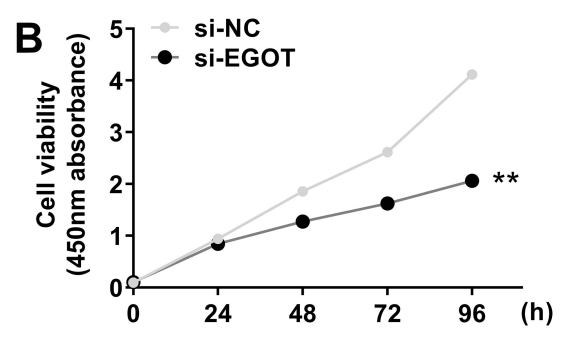

Si-EGOT

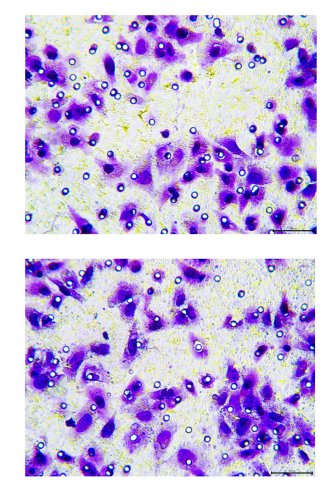

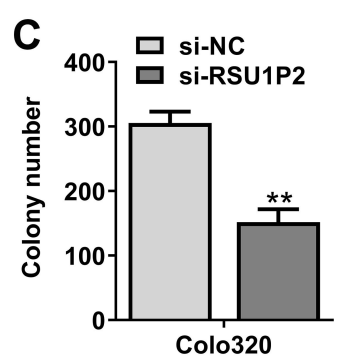

E
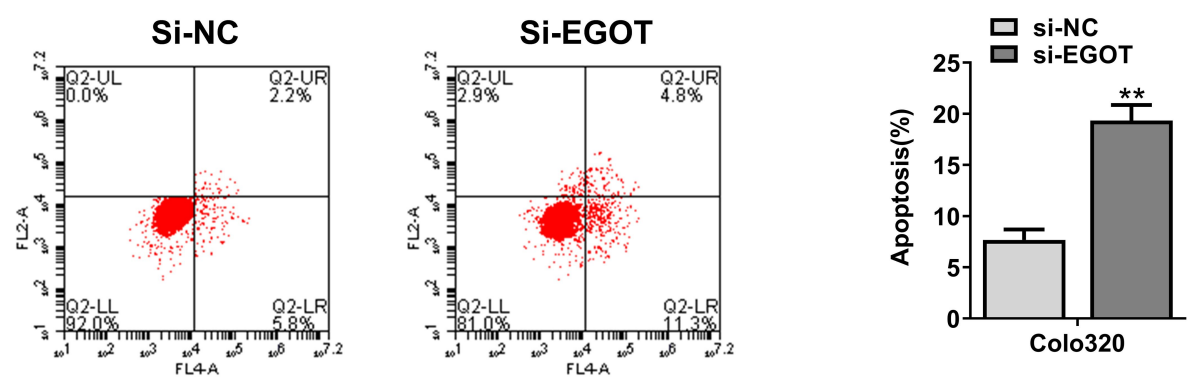

Figure 2 EGOT knockdown inhibited the growth of rectal cancer cells. (A) EGOT expression of siRNA EGOT-transfected Colo320 cells. (B) CCK-8 assay was used to assess cell viability. (C) Colony formation assay was used to assess cell colony formation. (D) Transwell was used to measure cell invasion and migration. (E) Flow cytometry was used to detect apoptosis. $\mathrm{N}=3$. $* * p<0.01$, $* * * p<0.00 \mathrm{I}$.

EGOT Affected Rectal Cancer Progression via the miR-2I I-5p/ErbB4 Axis

Next, the potential mechanism of miR-211-5p to regulate the progression of rectal cancer was explored. We predicted through online prediction tool Starbase v2.0 and ErbB4 was identified as a potential target for miR-211-5p (Figure 5A). The luciferase reporter gene assay showed that the luciferase activity of miR-211-5p mimic and ErbB4-WT co-transfected cells was significantly reduced $(\mathrm{P}<0.05)$, but the luciferase activity of ErbB4-MUT did not change significantly (Figure 5B). Compared with the miR-NC group, the mRNA and protein expression of ErbB4 in the miR-211-5p group was significantly reduced $(p<0.05)$, and the mRNA and protein expression of ErbB4 in the EGOT group was significantly increased $(p<0.05)$, while co-transfection of miR-211-5p and EGOT reversed the effect of miR-211-5p on the expression of ErbB4 $(p<0.05$, Figure 5C and D). As shown in Figure 5E, the expression of ErbB4 was significantly increased in rectal cancer tissues compared with Normal group $(p<0.05)(\mathrm{n}=50)$. In addition, as shown in Figure 5F, the expression of ErbB4 in rectal cancer tissue was negatively correlated with the expression of miR-211-5p $\left(\mathrm{R}^{2}=0.599\right)$. These results indicated that EGOT enhanced the expression of ErbB4 by regulating miR211-5p.

\section{The miR-2 II-5p Inhibitor Restored the Effect of EGOT Knockdown on}

\section{Radiotherapy}

Next, whether EGOT affected radiosensitivity through miR211-5p/ErbB4 axis was analyzed. As shown in Figure 6A and $\mathrm{B}$, the results of colony formation and apoptosis analysis showed that, compared with the si-NC group, both si-EGOT and 2Gy significantly inhibited cell proliferation $(p<0.05)$, while miR-211-5p inhibitor reversed the effect of si-EGOT on 

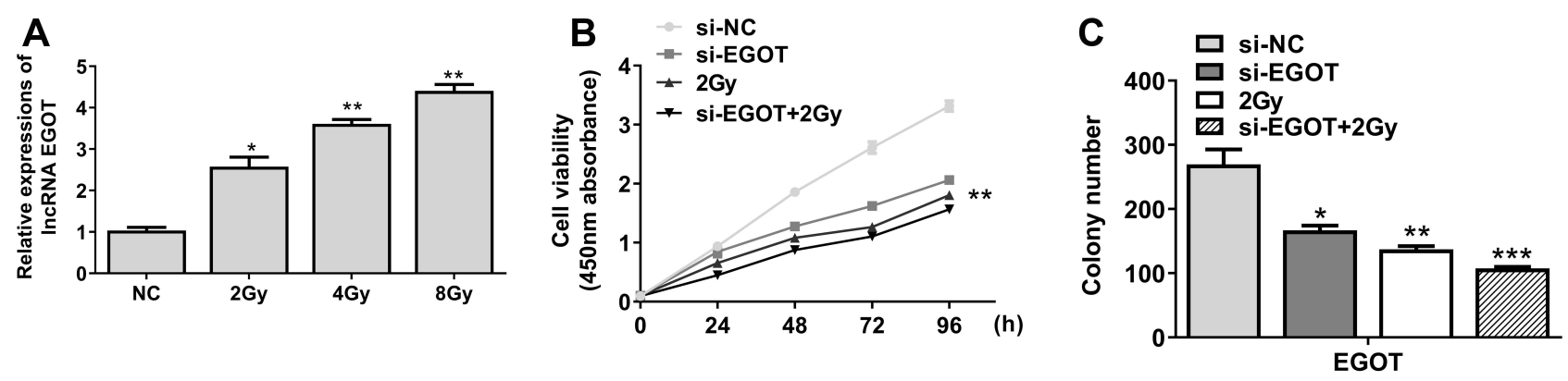

D
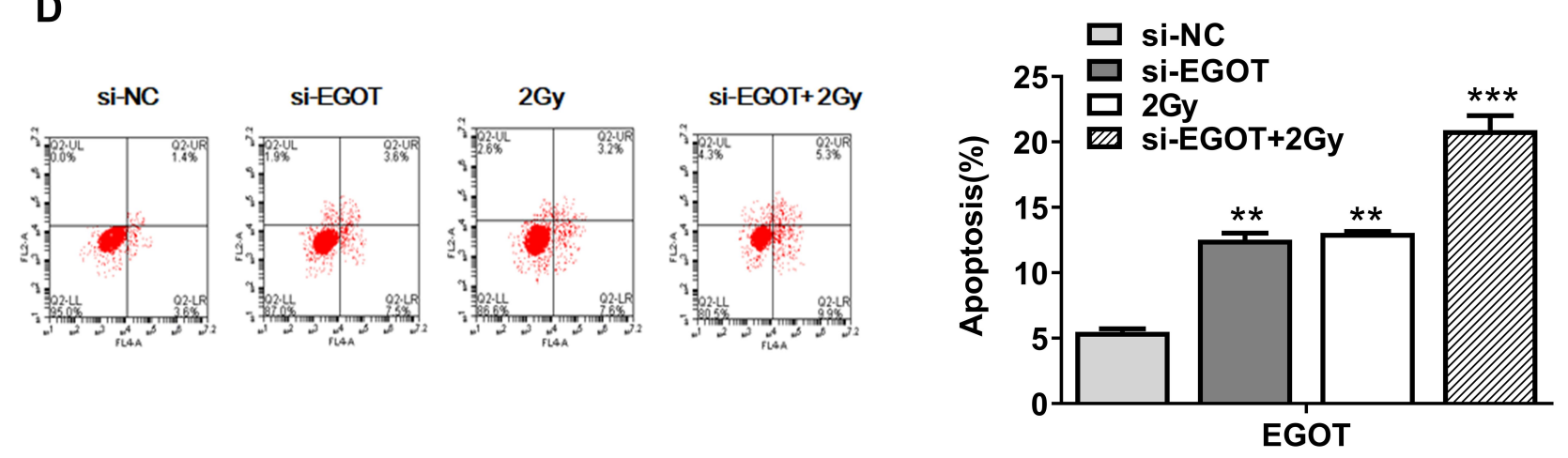

Figure 3 EGOT participated in radiotherapy for rectal cancer and affected radiosensitivity. (A) Expression level of EGOT in Colo320 cells. (B) CCK-8 was used to evaluate cell viability. (C) Colony formation test was used to evaluate the number of cell colonies formed. (D) Apoptosis was detected by flow cytometry. ${ }^{\mathrm{p}}<<0.5$, $* * p<0.0$, $* * * p<$ 0.001 .

$\begin{array}{lr}\text { A } & \\ & \\ \text { miR-211-5p } & \text { UUCCCUUUGUCAUCCUUCGCCU } \\ \text { EGOT } & ::::::::: \\ & \text { TCCCAGTTAGGAGGAGGCGC }\end{array}$

D

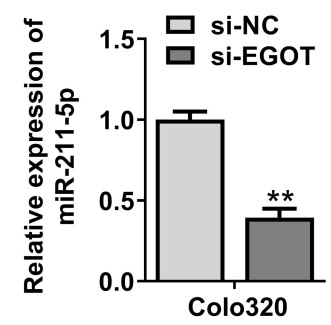

$\mathbf{B}$

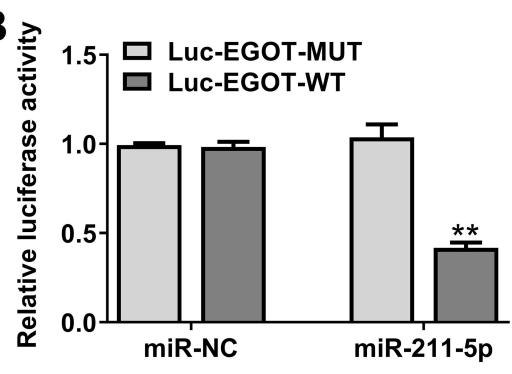

E

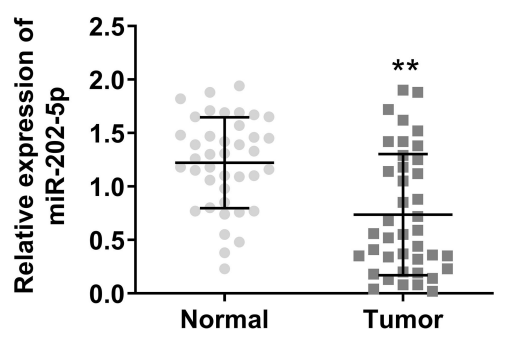

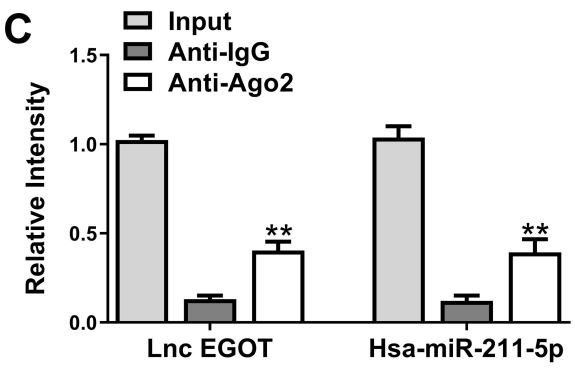

F

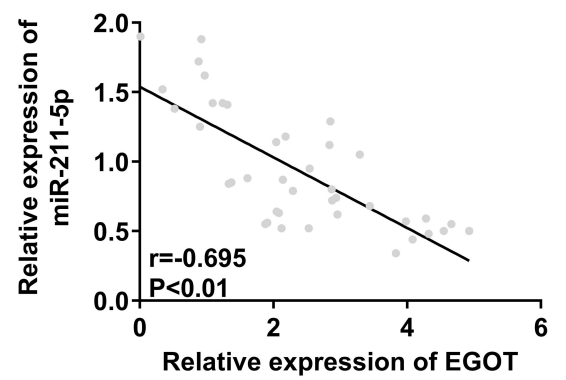

Figure 4 MiR-2I I-5p was a target of EGOT. (A) miR-2I I-5p was predicted to be a target gene of EGOT. (B) Fluorescein enzyme activity analysis in cells co-transfected with EGOT-WT or EGOT-Mut vectors. (C) Immunoprecipitation test was used to determine the enrichment of EGOT and miR-2II-5p in the immunoprecipitation complex. Antiimmunoglobulin G was used as a control. (D) Expression level of miR-2II-5p in EGOT-knockout Colo320 cells. (E) Expression level of miR-2II-5p in rectal cancer tissue. (F) Pearson correlation analysis of miR-2II-5p and EGOT in rectal cancer tissue $(n=40)$. N $=3$. **p $<0.01$. 
A

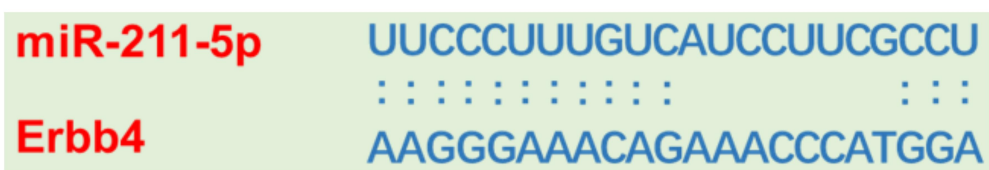

B

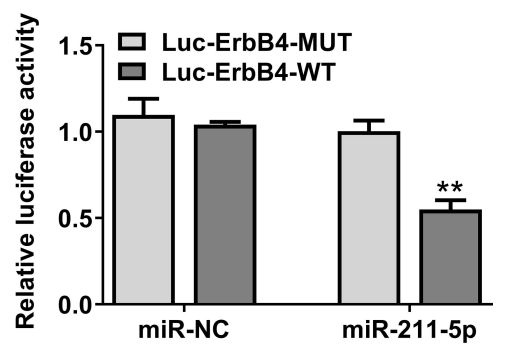

C

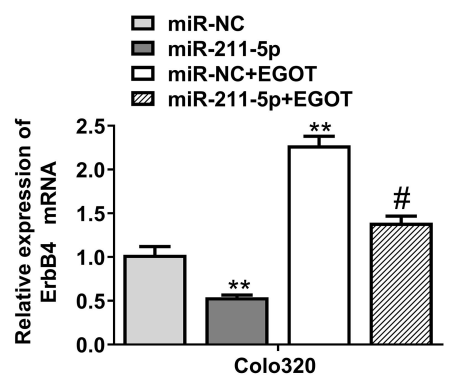

E

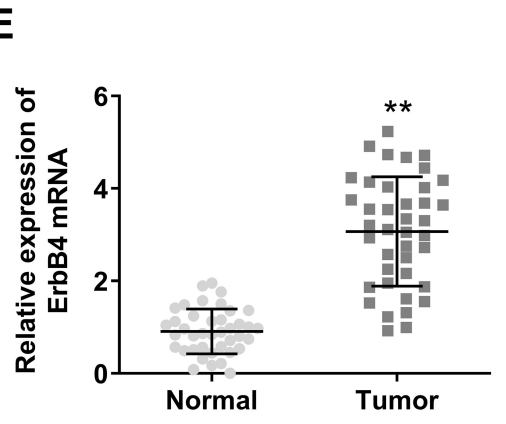

D

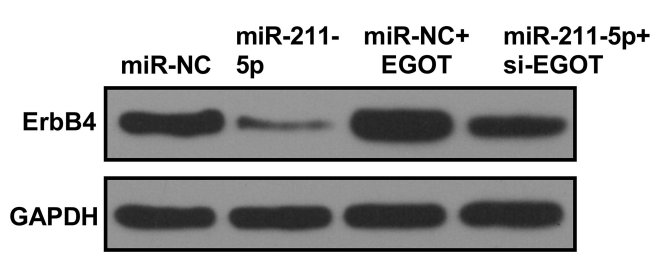

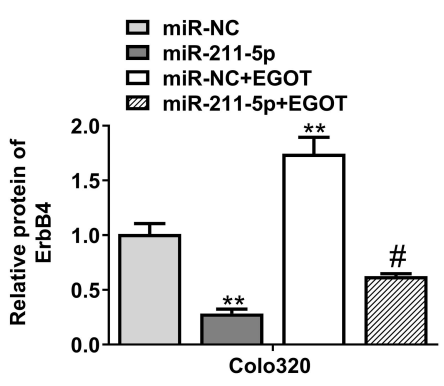

Figure 5 EGOT affected the progression of rectal cancer through the miR-2II-5p/ErbB4 axis. (A) ErbB4 was predicted to be a target gene of miR-2II-5p. (B) Analysis of luciferase activity in cells co-transfected with ErbB4-WT or ErbB4-Mut vectors. (C) ErbB4 mRNA expression level in Colo320 cells. (D) ErbB4 protein expression level in Colo320 cells. (E) ErbB4 expression level in rectal cancer tissue. $(\mathbf{F})$ Pearson correlation analysis of ErbB4 and miR-2II-5p in rectal cancer tissue $(\mathrm{n}=40)$. $\mathrm{N}=3$. $* * p<0.0 \mathrm{I}$ vs miR-NC; ${ }^{\#}<0.05$ vs miR-2II-5p.

radioactivity $(p<0.05)$. And as shown in Figure $6 \mathrm{C}$, the results of immunofluorescence staining of $\gamma-\mathrm{H} 2 \mathrm{AX}$ analysis showed that, compared with the si-NC group, both si-EGOT and $2 \mathrm{~Gy}$ significantly promoted average fluorescence intensity of $\gamma$ $\operatorname{H} 2 \mathrm{AX}(p<0.05)$, while miR-211-5p inhibitor reversed the effect of si-EGOT on radioactivity $(p<0.05)$. In addition, as shown in Figure 6D, compared with the si-NC group, si-EGOT could significantly decrease the protein expression level of ErbB4 $(p<0.05)$. As shown in Figure 6E, co-transfection of siEGOT with miR-211-5p inhibitor could reverse the effect of siEGOT on the protein level of ErbB4. These results indicated that the miR-211-5p inhibitor restored the effect of EGOT knockdown on radiotherapy.

\section{Inhibition of EGOT Enhanced Rectal}

\section{Cancer Radiosensitivity}

Finally, the effect of EGOT on the radiosensitivity of rectal cancer tumor tissues was analyzed. As shown in Figure 7A-C, compared with the control group, both shEGOT and 2Gy could significantly inhibit tumor growth rate, tumor volume and weight $(p<0.05)$, and combined treatment of sh-EGOT and 2Gy further inhibited tumor growth, tumor volume and weight $(p<0.05)$. And Ki-67 staining results showed that both sh-EGOT and 2 Gy could reduce the number of positive cells $(p<0.05)$, and combined treatment of sh-EGOT and 2 Gy further reduced the number of positive cells $(p<0.05$, Figure 7D). These 
A

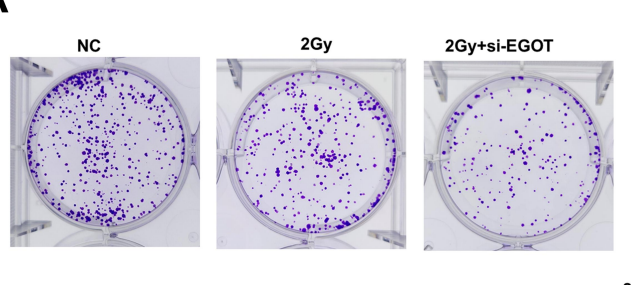

B
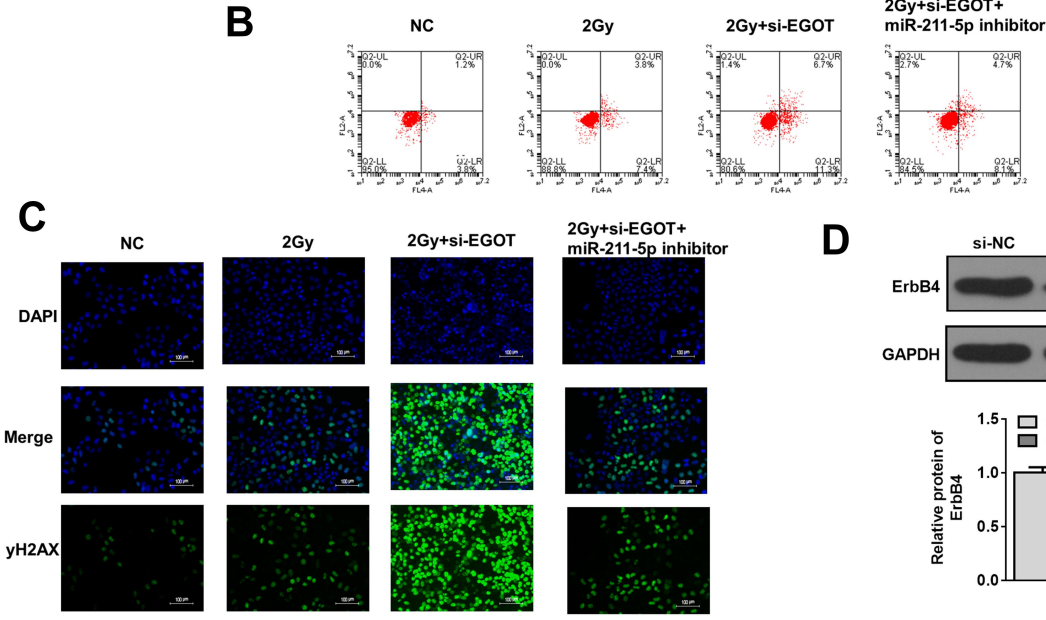

D
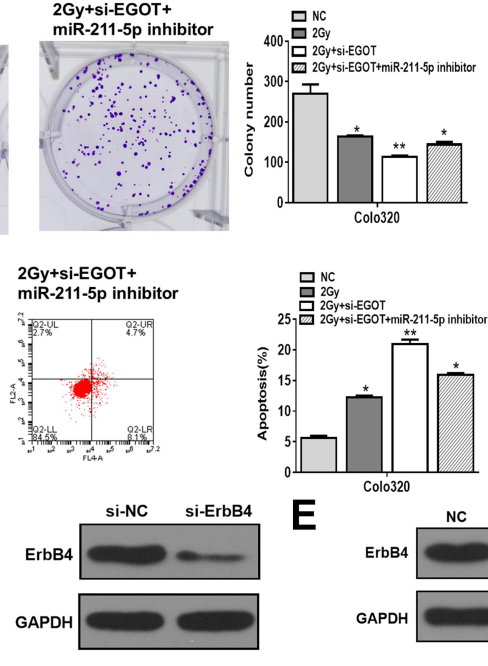

Figure 6 MiR-2I I-5p inhibitor restored the effect of EGOT knockdown on radiosensitivity. (A) Cell proliferation was evaluated by colony formation test. (B). Apoptosis was detected by flow cytometry. (C) Immunofluorescence detection of $\gamma-\mathrm{H} 2 \mathrm{AX}(\mathbf{D}$ and $\mathbf{E})$. The protein levels of ErbB4. $\mathrm{N}=3$. ${ }^{*} p<0.05$, $* * p<0.01$.

results indicated that the inhibition of EGOT enhanced rectal cancer radiosensitivity in vivo.

\section{Discussion}

Rectal cancer is still the fourth most common tumor in the world, and its mortality rate ranks third. ${ }^{26}$ Compared with other malignant tumors, rectal cancer is a heterogeneous disease with many causes, which has not yet been clarified. ${ }^{27}$ Radiation therapy is one of the main treatments for rectal cancer. Patients with the same clinical stage may have different results after receiving radiation therapy. The patients may have distant metastasis because of radiation tolerance and local lesions are not sensitive to radiotherapy, even threatening the life of patients. ${ }^{28}$ Therefore, it is of great significance to explore the regulatory mechanism of radiosensitivity of rectal cancer and to find potential therapeutic targets.

Radiation resistance of tumor tissues has been one of the main reasons for the failure of radiotherapy. ${ }^{29}$ Studies have shown that transcription factors and growth factor receptors involved in cell signal transduction, which affects radiosensitivity of tumor cells. ${ }^{30}$ LncRNAs have been gradually discovered by scholars, and its functions in cells have been gradually studied. ${ }^{31}$ LncRNA is strongly related to the aggressiveness, distant metastasis and prognosis of cancer patients. ${ }^{32}$ For example, IncRNA XIAPAs1 is highly expressed in colorectal cancer tissues, which not only promotes the proliferation function of colorectal cancer, but also can affect the invasion and metastasis of colorectal cancer by promoting the EMT process. ${ }^{33}$ It has been confirmed that IncRNA is associated with tumor radiosensitivity. For example, HOTAIR can induce radiation resistance in Hela cells by inhibiting p21, and lincRNA-p21 can increase the radiosensitivity by inducing apoptosis of colorectal cancer cells. ${ }^{34}$ However, no research has been reported on the radiosensitivity of IncRNA in rectal cancer. LncRNA EGOT is a recently discovered lncRNA. Studies have found that it is abnormally expressed in a variety of malignant tumors, and the expression of EGOT is up-regulated in glioma tissues. Consistently, this study found that EGOT was upregulated in rectal cancer tissues and cell lines, and overall survival in patients with high expression of EGOT was significantly reduced. These data indicated the potential carcinogenic effect of EGOT in rectal cancer. Si-EGOT could significantly reduce the viability of Colo320 cells, inhibit cell invasion, migration and induce apoptosis. In addition, irradiation doses of $2 \mathrm{~Gy}$ and increased the 


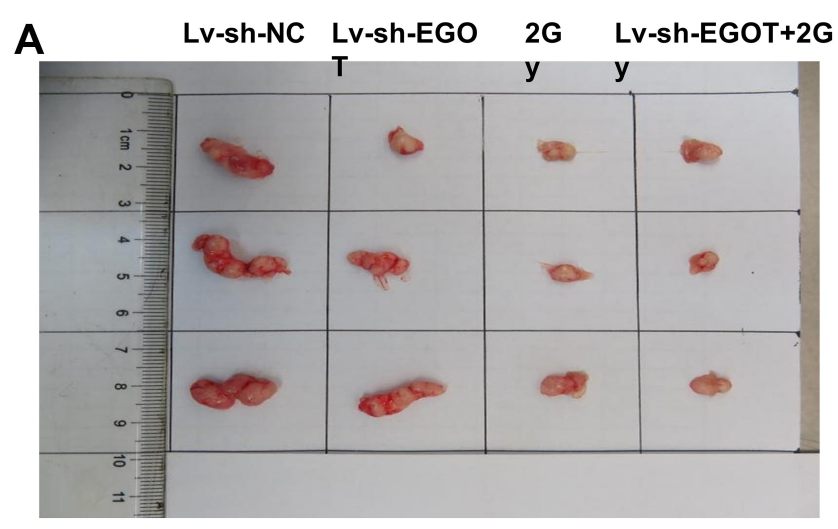

B

C
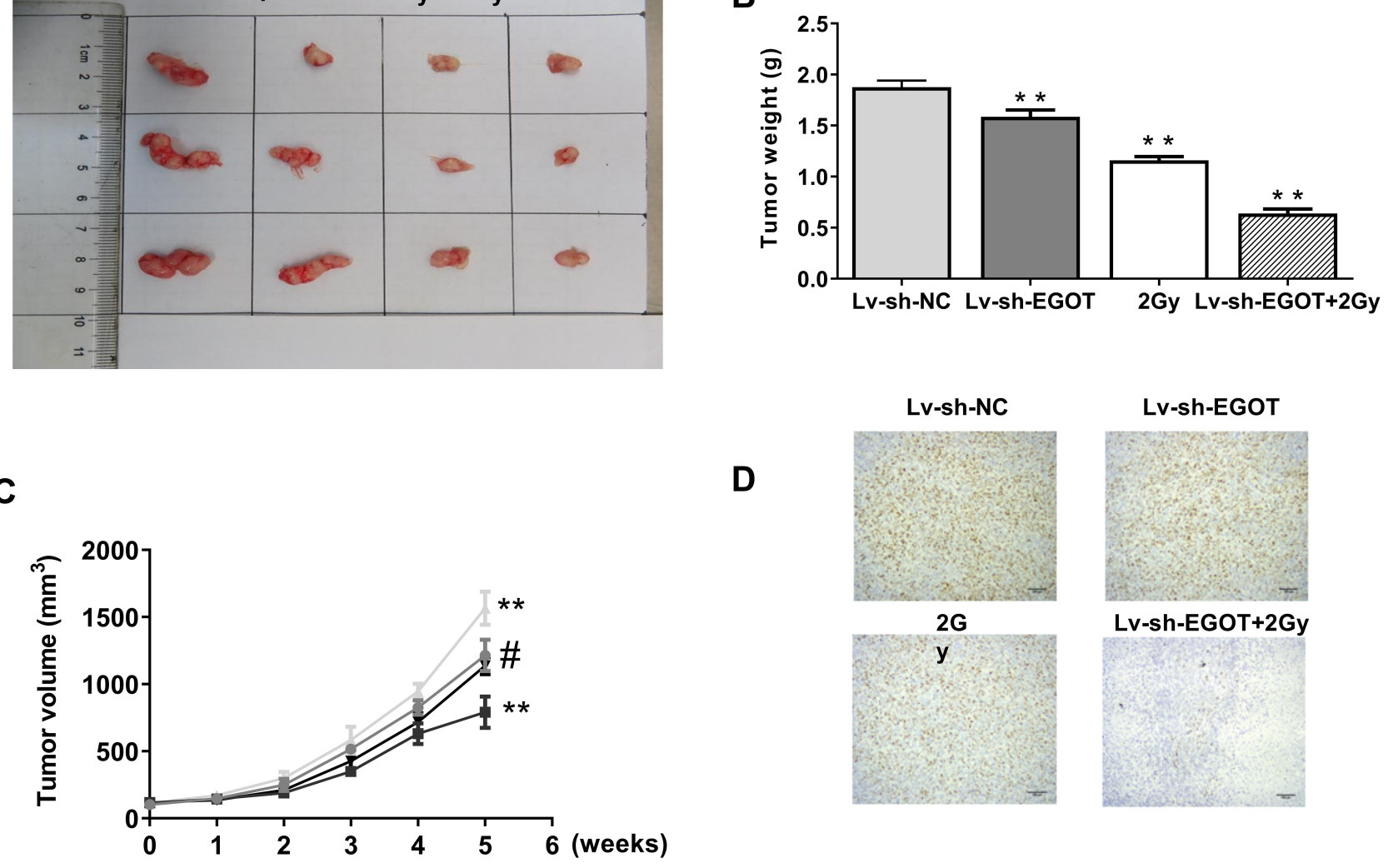

D

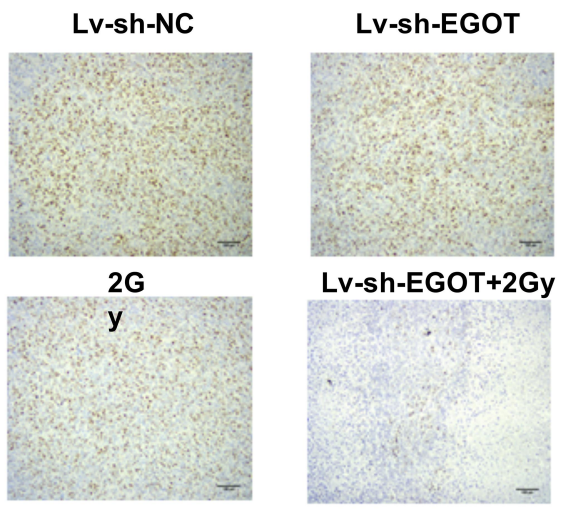

Figure 7 EGOT knockdown enhanced the radiosensitivity of rectal cancer. (A) Measurement of tumor volume in nude mice weekly. (B) Comparison of tumor weight in nude mice. (C) Representative images of three groups of subcutaneous tumors. (D) Ki67 staining. N= 5. ${ }^{* *} p<0.01$ vs Lv-sh-NC; ${ }^{\#} p<0.05$ vs Lv-sh- EGOT.

expression of EGOT or the combined treatment of siEGOT and 2Gy could further inhibited cell proliferation, induced apoptosis and inhibited tumor growth in vivo. These results indicated that EGOT was involved in radiotherapy for rectal cancer and affected radiosensitivity.

LncRNA can be used as a sponge for miRNA, targeting mRNA to promote cell proliferation and apoptosis. ${ }^{35}$ miRNA plays an important role in different cell activities such as proliferation and apoptosis of biological cells. In the process of carcinogenesis and development of rectal cancer, miRNA can not only promote tumorigenesis by regulating the expression of oncogenes, but also inhibit tumorigenesis by inhibiting the expression of oncogenes. ${ }^{36}$ For example, miRNA has been found that miR-29c-3p is expressed at a low level in rectal cancer tissues and cell lines, and it plays an important role in the regulation of radiosensitivity. ${ }^{37}$ Studies have shown that miR-521 regulates the expression of the DNA repair protein CSA, and makes prostate cancer cells sensitive to radiation therapy. ${ }^{38}$ The overexpression of miR-181a makes malignant glioma cells sensitive to radiation therapy and down- regulates the Bcl-2 protein. ${ }^{39}$ MiR-211-5p is a miRNA with tumor growth inhibitory effects. Studies have found that miR-211-5p regulates tongue cancer cell proliferation and cisplatin resistance through Ezrin/Fak/Src signal pathways. And studies have found that miR-211-5p may be a tumor suppressor in the tumorigenesis of renal cell carcinoma (RCC) and may be a potential therapeutic target for RCC in the future. Further research should focus on the underlying mechanism of miR-211-5p in RCC and on investigating the possible use of miR-211-5p as a biomarker for RCC. ${ }^{20}$ MiR-211-5p was screened as a target gene of EGOT. MiR-211-5p was down-regulated in rectal cancer tissues, and miR-211-5p inhibitor could reverse the effects of si-EGOT on radioactivity. These results indicated that EGOT regulated sensitivity of colorectal cancer cells through miR-211-5p.

ErbB4 is the fourth member of the ErbB family to be discovered. ErbB4 is over-expressed in many tumors and is closely related to uncontrolled growth and proliferation of tumor cells, as well as infiltration and metastasis. ${ }^{40}$ Studies have shown that transfection of ErbB4 gene can enhance cell 
proliferation ability, while ErbB4 monoclonal antibody can inhibit the proliferation rate and increase the apoptosis rate. Knockout ErbB4 can inhibit the migration and invasion of esophageal squamous carcinoma cell lines. ${ }^{23}$ MiR-302b can down-regulate the expression of ErbB4 after transcription, which induces the apoptosis of esophageal squamous carcinoma cell lines and inhibits its invasiveness. ${ }^{41}$ Studies have found that in three derivative clones of the transfected H1299 cells demonstrated greater growth in comparison with parental cells. They have also increased resistance to several drugs but their radiosensitivity was not changed. These results supported the hypothesis that ErbB-4 played a significant role in tumor growth and in the tumor cell resistance to chemotherapy. ${ }^{42}$ In this study, ErbB4 was screened as a target gene of miR-211-5p through database. The expression of ErbB4 was up-regulated in rectal cancer tissues and significantly decreased after miR-211-5p group. The expression of ErbB4 was significantly increased in the EGOT group. Co-transfection of miR-211-5p with EGOT reversed the effect of miR-211-5p on the expression of ErbB4. $\gamma-\mathrm{H} 2 \mathrm{AX}$ is considered as an important biomarker for detection of DNA double-strand breaks. $\gamma$-H2AX immunofluorescence method, which uses specific fluorescent antibody labeling technology of $\gamma-\mathrm{H} 2 \mathrm{AX}$ and fluorescence microscope, is the most important method to study $\gamma$ $\mathrm{H} 2 \mathrm{AX}$, and it is also a reliable detection method developed in recent years to detect DSBs caused by low dose radiation in vivo and in vitro. ${ }^{43}$ The results of immunofluorescence staining of $\gamma-\mathrm{H} 2 \mathrm{AX}$ analysis showed that both si-EGOT and $2 \mathrm{~Gy}$ significantly promoted average fluorescence intensity of $\gamma$-H2AX compared with that in the NC group, while miR211-5p inhibitor reversed the effect of si-EGOT on radioactivity. These results indicated that EGOT knockdown increased radiosensitivity of rectal cancer by targeting miR211-5p/ErbB4 axis.

\section{Conclusion}

EGOT knockdown could inhibit the proliferation of rectal cancer cells by regulating the miR-211-5p/ErbB4 axis and improve the radiosensitivity of rectal cancer by targeting miR-211-5p/ErbB4 axis. This might provide a new field of vision for the diagnosis and treatment of rectal cancer.

\section{Funding}

This work was supported by National Natural Science Foundation of China (81972196), National Key Research and Development Program of the Ministry of Science and
Technology of China (2016YFC1303201, 2016YFC0905 300, 2018YFC1315000), Non-profit Central Research Institute Fund of Chinese Academy of Medical Sciences (2018PT32012), CAMS Innovation Fund for Medical Sciences (CIFMS) (2017-I2M-1-006, 2019-I2M-2-002), and Beijing Hope Run Special Fund from Cancer Foundation of China (LC2017A19).

\section{Disclosure}

All the authors declare that they have no conflict of interests for this work.

\section{References}

1. Liang K, Yuan-Guang C, Hao Z, et al. Transanal total mesorectal excision for rectal cancer: a multicentric cohort study. Patterns. 2020;1:1. doi:10.1016/j.patter.2020.100057

2. Arja V, Mehran G, Ries K, Chemistry S. Estimation of prostate cancer probability by logistic regression: free and total prostate-specific antigen, digital rectal examination, and heredity are significant variables. Clin Chem. 2020;7:7.

3. Wang R, Fan Q, Liu H, Medicine M. Isolated vaginal recurrence of early-stage rectal cancer detected by $18 \mathrm{~F}-\mathrm{FDG}$ PET/CT. Clin Nucl Med. 2019;44(6):1.

4. Keller DS, Paula T, Kiran RP. Ready for the national accreditation programs for rectal cancer? Auditing rectal cancer outcomes in the United States. Colorectal Dis. 2019;21:1213-1215. doi:10.1111/codi.14729

5. Lee GC, Bordeianou LG, Francone TD, Blaszkowsky LS, Endoscopy MQ. Superior pathologic and clinical outcomes after minimally invasive rectal cancer resection, compared to open resection. Surg Endoscopy. 2019;34:3435-3448. doi:10.1007/ s00464-019-07120-2

6. Khorasani S, Nagarajan A, Nguyen T, Chadi SA. Principles of adjuvant and neoadjuvant therapy for locally advanced rectal cancer. Fundam Anorectal Surg. 2019.

7. Parikh K, DeNittis AS, Marks G, Zeger E, Oncology J. Neoadjuvant chemotherapy and high-dose radiation using intensity-modulated radiotherapy followed by rectal sparing TEM for distal rectal cancer. J Radiation Onco. 2019;8(2):217-224.

8. Li -C-C, Liang J-A, Chen W, Chien C-R. Effectiveness of image-guided radiotherapy for rectal cancer patients treated with neoadjuvant concurrent chemoradiotherapy: a population-based propensity score-matched analysis. Asia-Pacific J Clin Oncol. 2019;15. doi:10.1111/ajco.13196

9. Hu H, Yu X, Zhou J. Long noncoding RNA MALAT1 enhances the apoptosis of cardiomyocytes through autophagy modulation. Biochem Cell Biol. 2020;98(2):130-6

10. Yang L, Li L, Zhou Z, Liu Y, International S. SP1 induced long non-coding RNA LINC00958 overexpression facilitate cell proliferation, migration and invasion in lung adenocarcinoma via mediating miR-625-5p/CPSF7 axis. cancer cell int. 2020;20:1.

11. Farhana M, Varinder J, Clements JA, Yousef GM, Jyotsna B. MicroRNA theranostics in prostate cancer precision medicine. clin chem. 2020;10:10.

12. Xue Y, Ni T, Jiang Y, Li Y. Long noncoding RNA GAS5 inhibits tumorigenesis and enhances radiosensitivity by suppressing miR-135b expression in non-small cell lung cancer. Oncol Res. 2017;25:8.

13. Kumar M, Devaux RS, Shen JJ, Davis SP, Herschkowitz JI. Abstract 1598: IncRNA AK001796 as a therapeutic target in aggressive breast cancers. Cancer Res. 2016;76(14 Supplement):1598. 
14. Jin L, Quan J, Pan X, et al. Identification of lncRNA EGOT as a tumor suppressor in renal cell carcinoma. Mol Med Rep. 2017;16 (5):7072-7079. doi:10.3892/mmr.2017.7470

15. Peng W, Wu J, Fan H, Lu J, Feng J, Research O. LncRNA EGOT promotes tumorigenesis via hedgehog pathway in gastric cancer. Pathol Oncol Res. 2017;25(1):1-5.

16. Xiao B, Zhang W, Chen L, et al. Analysis of the miRNA-mRNAIncRNA network in human estrogen receptor-positive and estrogen receptor-negative breast cancer based on TCGA data. Gene. 2018;658:28-35. doi:10.1016/j.gene.2018.03.011

17. Emma R, Niku O, Terho L. MicroRNAs in the atherosclerotic plaque. Clin Chem. 2020;12:12.

18. Nerea M, Teresa VM, Ricardo GC, et al. Tumor MicroRNA expression profiling identifies circulating MicroRNAs for early breast cancer detection. Clin Chem. 2020;8:8.

19. Li T, Ma J, Han X, et al. MicroRNA-320 enhances radiosensitivity of glioma through down-regulation of sirtuin type 1 by directly targeting forkhead box protein M1. Transl Oncol. 2018;11(2):205-212. doi:10.1016/j.tranon.2017.12.008

20. Quan J, Pan X, He T, et al. Tumor suppressor miR-211-5p is associated with cellular migration, proliferation and apoptosis in renal cell carcinoma. Exp Ther Med. 2018. doi:10.3892/etm.2018.5908

21. Díazmartínez M, Benitojardón L, Alonso L, Koetzploch L, Hernando E, Teixido J. miR-204-5p and miR-211-5p contribute to BRAF inhibitor resistance in melanoma. Cancer Res. 2018;78(4):1017.

22. Shin-ichi F, Tadao F, Hideo H, et al. Quantitative analysis of a MDR1 transcript for prediction of drug resistance in acute leukemia. Clin Chem. 2020;6:6.

23. Murakami A, Nakamura M, Kaneko S, Lin WL, Kusaka H. Aberrant accumulation of ErbB4 in progressive supranuclear palsy. Neuropathol Appl Neurobiol. 2018;44:6. doi:10.1111/nan.12460

24. Liu Q, Cornejo KM, Cheng L, Hutchinson L, Jiang Z. Nextgeneration sequencing to detect deletion of RB1 and ERBB4 genes in chromophobe renal cell carcinoma: a potential role in distinguishing chromophobe renal cell carcinoma from renal oncocytoma. $\mathrm{Am} \mathrm{J}$ Pathol. 2018;188:4.

25. Sanaa E, Seada L. Quantitation of bcl-2 protein in bladder cancer tissue by enzyme immunoassay: comparison with Western blot and immunohistochemistry. Clin Chem. 2020;7:7

26. Valentini V, Schmoll H, Velde J. Multidisciplinary management of rectal cancer: questions and answers. oncol hematol. 2018.

27. Caycedo-Marulanda A, Jiang HY, Kohtakangas E. Outcomes of a single surgeon-based transanal-total mesorectal excision (TATME) for rectal cancer. $J$ Gastrointest Cancer. 2018;49(4):455-462. doi:10.1007/s12029-017-9989-7

28. Das P, Minsky BD. Radiation therapy for rectal cancer. rec cancer. 2018.

29. Chang X, Xue X, Zhang Y, Zhang G, Liu H. The role of NRAGE subcellular location and epithelial-mesenchymal transition on radiation resistance of esophageal carcinoma cell. $J$ Cancer Res Ther. 2018;14(1):46.
30. Ma J, Lu Y, Zhang S, Li Y, Xu S. Differentiation, $\beta$-Trcp ubiquitin ligase and RSK2 kinase-mediated degradation of FOXN2 promotes tumorigenesis and radioresistance in lung cancer. cell death differ. 2018;25(8).

31. Wan Y, Cycle Z-Q. LncRNA NEAT1 affects inflammatory response by targeting miR-129-5p and regulating Notch signaling pathway in epilepsy. cell cycle. 2020;11:1-13.

32. Lorenzen JM, Celina S, Malte $\mathrm{K}$, et al. Long noncoding RNAs in urine are detectable and may enable early detection of acute $\mathrm{T}$ cellmediated rejection of renal allografts. Clin Chem. 2020;12:12.

33. Zheng Z, Zhang X, Wang J, et al. Exposure to fine airborne particulate matters induces hepatic fibrosis in murine models. $J$ Hepatol. 2015;63(6):1397-1404. doi:10.1016/j.jhep.2015.07.020

34. Wu X, Cao XB, Chen F. WITHDRAWN: IncRNA-HOTAIR activates tumor cell proliferation and migration by suppressing MiR-326 in cervical cancer. Oncol Res. 2019.

35. Liu Z, Xu S, Dao J, Gan Z, Physiology X. Differential expression of lncRNA/miRNA/mRNA and their related functional networks during the osteogenic/odontogenic differentiation of dental pulp stem cells. Mol Cancer. 2019;2.

36. Cui S, Cao Z, Guo W, Yu H, University Y. Plasma miRNA-23a and miRNA-451 as candidate biomarkers for early diagnosis of nonsmall cell lung cancer: a case-control study. J South Med Univ. 2019;39 (6):705-711. doi:10.12122/j.issn.1673-4254.2019.06.12

37. Wang J, Xu J, Fu J, et al. MiR-29a regulates radiosensitivity in human intestinal cells by targeting PTEN gene. Nan Fang Yi Ke Da Xиe Xue Bao. 2016;186(3):292.

38. Liu Z, Liang $X$, Li X, et al. MiRNA-21 functions in ionizing radiation-induced epithelium-to-mesenchymal transition (EMT) by downregulating PTEN. Radiat Res. 2019;8.

39. Shen H, Wang L, Xiong J, Ren C. Long non-coding RNA CCAT1 promotes cervical cancer cell proliferation and invasion by regulating the miR-181a-5p/MMP14 axis. Toxicol Res (Camb). 2019;18 (10): $1-12$

40. Lin TW, Tan Z, Barik A, et al. Regulation of synapse development by Vgat deletion from ErbB4-positive interneurons. Cell Cycle. 2018;38 (10):617669.

41. Wang N, Zhang Z, Lv J. Fentanyl inhibits proliferation and invasion via enhancing miR-302b expression in esophageal squamous cell carcinoma. Oncol Letters. 2018. doi:10.3892/ol.2018.8616

42. Vexler A, Starr A, Gladish V, et al. The role of ErbB-4 expression in non-small cell lung cancer (NSCLC) in cell growth and sensitivity to chemo- and radiotherapy. Cancer Res. 2004;64.

43. Turner HC, Brenner DJ, Chen Y, Bertucci A, Garty G. Adapting the $\gamma-\mathrm{H} 2 \mathrm{AX}$ assay for automated processing in human lymphocytes. 1 . Technological aspects. Radiat Res. 2011;175(3):282-290. doi:10.1667/RR2125.1
OncoTargets and Therapy

\section{Publish your work in this journal}

OncoTargets and Therapy is an international, peer-reviewed, open access journal focusing on the pathological basis of all cancers, potential targets for therapy and treatment protocols employed to improve the management of cancer patients. The journal also focuses on the impact of management programs and new therapeutic agents and protocols on patient perspectives such as quality of life, adherence and satisfaction. The manuscript management system is completely online and includes a very quick and fair peer-review system, which is all easy to use. Visit http://www.dovepress.com/ testimonials.php to read real quotes from published authors. 